\title{
Understanding the Polymerization Process of Eumelanin by Computer Simulations
}

\author{
Aleandro Antidormi, ${ }^{, \dagger}{ }^{\dagger}$ Claudio Melis, ${ }^{\dagger}$ Enric Canadell, ${ }^{\ddagger}$ and Luciano Colombo ${ }^{\dagger}$ \\ $\dagger$ †ipartimento di Fisica, Università di Cagliari, Cittadella Universitaria, I-09042 \\ Monserrato (Ca), Italy \\ $\ddagger$ Institut de Ciència de Materials de Barcelona (ICMAB-CSIC), Campus de Bellaterra, \\ 08193 Bellaterra, Barcelona, Spain \\ E-mail: aleandro.antidormi@dsf.unica.it
}

\begin{abstract}
We present and discuss a computational setup aimed at modelling the polymerization process of eumelanin by blending together molecular dynamics simulations and ab-initio total energy calculations. By simulating the oxidative polimerization process of eumelanin in methanol we provide evidence that "in silico" sinthesized samples are mostly composed by small-weight molecular structures: an exponential distribution of the molecular weights is found, yielding an average weight smaller than 10. Adopting the scheme of chemical disorder, we also perform a statistical analysis on the variety of molecules formed, focusing on both their structural and electronic features. In particular, 1) we identify the most frequent structural patterns within the molecules by analyzing the occurrence of the single chemical bonds. Moreover, 2) we show how most of the molecules have a large number of non-terminated Oxygen atoms, highlighting the pivotal role of $=\mathrm{O}$ groups in the eumelanin molecular stability.
\end{abstract}




\section{Introduction}

Eumelanin is a biological pigment present in animals, plants and microorganisms mostly known to perform active functions in the human pigmentary cells, like skin coloration and photoprotection against exposure to UV radiation. ${ }^{1-3}$ In particular, ion-exchange as well as the pro-/anti-oxidant activities and electrical transmission are recognized to be prominent features of the eumelanin macromolecules. These properties have recently attracted the interest of scientists from different disciplines toward the exploitation of synthetic eumelanin in several applications, from organic electronics ${ }^{2}$ to biointerfaces ${ }^{4,5}$ and hybrid materials ${ }^{6}$ to polymer stabilization ${ }^{7}$ and photovoltaics. ${ }^{8,9}$

These achievements are unfortunately made difficult by the fact that the actual structure

of its molecule is mostly undefined; ${ }^{10-12}$ its determination has indeed encountered considerable difficulties primarily due to the pigment amorphous character and insolubility in all solvents.

Based on the investigation efforts made to-date, there is nonetheless a general agreement in that all the synthetic eumelanin molecules derive from the 5,6-dihydroxy-indole (DHI) monomer and its redox forms (see figure 1); these basic components can covalently bond to form oligomers ${ }^{13}$ (dimers, tetramers, pentamers and so on), known as the protomolecules. Several plausible protomolecular structures have been proposed in the recent literature, aimed at explaining the broad optical absorption spectrum in biological systems. ${ }^{14,15}$

A viewpoint which has recently gained credit is that eumelanin properties could be suitably described in terms of a "chemical disorder" model, not prescribing a specific molecular configuration, but considering eumelanins as a collection of structurally diverse molecular species. ${ }^{2,16}$ In this respect, eumelanin properties might descend from the aggregation of many different molecular configurations differing in both the structure and the oxidation state. Such a theoretical framework, which has proved to successfully reproduce the broad absorption spectrum of eumelanin ${ }^{15}$, points at the fundamental role played by the polymerization process: the variety of molecular configurations is univocally determined by the 
chemical processes occurring in the synthesis phase. This scenario has motivated the need of investigating the oxidative polymerization of the monomeric building blocks, whose detailed chemical events remain largely not understood.

Some experimental studies have been nevertheless able to show that: (i) the protomolecules of the most common forms of eumelanin produced in laboratory have small molecular weight; ${ }^{14,17-19}$ (ii) the reaction mechanism underlying the eumelanin polymerization encompasses single oxidative-coupling steps in the polymer elongation process. ${ }^{20,21}$ More specifically, the formation of oligomers starts with a dimerization step involving a DHI monomer and one of its oxidized forms, followed by subsequent coupling reactions increasing the size of the molecules. It has been argued that such a coupling mechanism results in the formation of non-oxidized oligomers, i.e. consisting of uniquely DHI units. Eventual oxidation processes can also occur which modify the electronic configurations of the created molecules. Figure 2 illustrates the basic oxidation and coupling: in (a) a DHI monomer transforms into its IQ redox form by releasing two hydrogen atoms (note that the oxidation can occur through several different and often not well known ways. Here we describe this process with a formal single reaction in which two $\mathrm{H}$ are released); (b) depicts an example of dimerization process in which a DHI unit bonds with an IQ monomer to form a 2,4'-dimer (in its non-oxidized form).

This notwithstanding, concerns still exist on the actual oligomer size distribution and on the structural complexity of the protomolecules in experimentally synthesized samples, being quantities hardly accessible from real laboratory measurements. In particular, it is important to explore the prevalent bonding patterns and the corresponding conformational implications, from which the macroscopic physico-chemical properties of eumelanin might follow.

Atomistic simulations may prove useful to offer new relevant information on this issue, since they could provide monomer-level insight into the polymerization process on one side, and allow for a microscopic characterization of the polymerized samples on the other. 
To this aim, we worked out a novel computational approach to model eumelanin polymerization which allows to reproduce "in silico" the chemical processes leading from monomeric units to a polymerized sample. Based on first-principles calculations blended to classical molecular dynamics, our tool allows to follow the evolution of systems made of thousands of atoms yet mantaining an accurate description of the ongoing chemical processes. Here we investigate (i) the eumelanin aggregates in terms of the average oligomer size and the corresponding size distribution and (ii) the configurational and electronic variety of the created oligomeric molecules.

The paper is organized as follows. In the next section we will describe the computational tool that we elaborated to reproduce the eumelanin polymerization process "in silico". Then the results of the numerical simulations will be discussed. In particular, the system under study will be detailed and the configurational and electronic analysis of the molecules will be given. Conclusions will finally be drawn.

\section{Computational Methods}

The numerical simulation of systems undergoing a polymerization process represents an appealing yet complex scientific task whose implementation requires three fundamental aspects to be properly described, namely: i) polymerization is a process taking place in systems with macroscopic dimensions, generally involving a very large number of molecular components; ii) it is a dynamical process determined by phenomena occurring on very different time scales: from chemical reactions, which occur on time scales of a few femtoseconds, to purely diffusive phenomena which could take from hours to days; iii) the fundamental steps responsible for the polymer elongation are represented by the creation of chemical bonds whose description should in principle require a quantum-mechanical treatment. A fully quantum-mechanical description of the dynamical evolution of large molecular systems would clearly satisfy the previous requirements but it is practically unfeasible; as a consequence, computational tools 
have been created to-date which employ a phenomenological approach to treat the chemical reactions and/or give up the atomistic description of the molecular components. ${ }^{22-25}$ In order to overcome such limitations, we propose a novel approach where molecular dynamics (MD) simulations are blended with first-principle total-energy calculations, so as to generate a fully atomistic modelling of polymerization. MD takes charge of the dynamical evolution of the system for very long time periods whereas first-principles calculations are exploited to describe (in a probabilistic approach) the creation of chemical bonds and the molecular oxidation processes. Specifically, we model the polymerization of eumelanin through a three-step procedure, faithfully replicating the polymerization scheme experimentally identified (figure 2). Such steps are iteratively repeated until the polymerized sample has been achieved. They are a) the "dynamical evolution", b) the "oxidation" and c) the "coupling" and will be described in detail in the following sections.

\section{Step 1: Dynamical evolution}

The first step is responsible for the dynamical evolution of the oligomeric components, calculating the trajectory of all the atoms in the system. The positions and velocities of the interacting components are evolved according to Newton's equations of motion, as implemented in the LAMMPS ${ }^{26}$ molecular dynamics package. To describe the inter- and intramolecular interactions in eumelain molecules, we used the AMBER force field ${ }^{27}$ which had been already successfully employed to model interactions in other polymeric structures ${ }^{28}$ and includes both bonding (bonds, bending, torsional) and nonbonding (van der Waals plus Coulomb) contributions. The parameters in the bonding and VdW terms are taken from the GAFF database. ${ }^{29}$ The velocity-Verlet algorithm with a time step of 0.5 fs is used to solve the equations of motion. A particle-particle particle-mesh solver is used for describing the long-range electrostatic forces, and the van der Waals interaction are cut off at $0.1 \mathrm{~nm}$. The Nose-Hoover thermostat and barostat with corresponding relaxation time equal to 50 fs and 0.5 ps respectively are used to control the simulation temperature and pressure. 


\section{Step 2: Coupling}

The formation of a chemical bond between oligomers is accomplished at this step by adopting a probabilistic approach. To this aim, we introduce a "bond creation probability" $p$, defined as the probability that two units can bond together forming a larger oligomer. At each time step, the distance between any pair of oligomeric units is checked and whether smaller than a given threshold ( $3 \AA$ in our case), the formation of a new bond is proclaimed according to the relative probability. The corresponding terms in the classical interaction potential are then accordingly updated with the introduction of new bonds, angles and dihedrals. Once this step has been performed, the procedure continues with a new iteration of the dynamical evolution.

In our model, we assume that different probability values $p$ correspond to different chemical reactions according to

$$
p=\min (1, \exp (\Delta E /(k T))
$$

where $k$ is the Boltzmann constant, $T$ is the temperature and $\Delta E$ is the difference between the energy of the reaction products and that of the reagents. The bond is then formed with probability 1 if the corresponding reaction is energetically favourable, otherwise it is created with a probability proportional to the Boltzmann weight. We remark that two units can differ in their size, oxidation state and for their linking sites, hence resulting in a very large number of possible different reactions (and probability values). Nevertheless, since

product analysis of different experimental works designed to isolate oligomers ${ }^{2,30}$ suggests that the coupling step involves the nucleophilic attack of the indole system to an oxidized electrophilic unit, we will consider only bonding mechanisms in which one DHI unit bonds with one oxidized form to create a non-oxidized molecule.

The values of $\Delta E$ in eq. (1) represent the main ingredient in our probabilistic model and contain the chemical aspect of the overall polymerization process. They are estimated using first-principle calculations using Density-functional theory (DFT) calculations as im- 
plemented in the plane-wave Quantum Espresso package. ${ }^{31}$ The Perdew-Burke-Ernzerhof (PBE) exchange-correlation functional ${ }^{32}$ is used together with ultrasoft pseudopotentials ${ }^{33}$ and Grimme correction to include dispersive forces in the system. ${ }^{34,35}$ We used energy cutoffs of 20 Ry and 250 Ry for the wavefunction and electronic density, respectively. Structures have been relaxed until all the atomic forces were smaller than 0.001 a.u. Periodic boundary conditions are applied to this large supercell with the Brillouin zone sampled at the $\Gamma$ point only.

Due to the extremely large number of possible reactions, the bond creation probability of a reaction involving oligomers with arbitrary sizes is estimated only considering the reaction between the effectively bonding monomeric units. In doing so, we are assuming a "local interaction approximation" according to which the energetics governing the reaction is essentially the one of the only interacting units. It is worthy to mention that in our computational approach the chemical reactions in which the breaking of a chemical bond occurs are consistently described by the very same formula (1) with the correct identification of the products and reagents energies.

Importantly, our model is also capable of including the effect of solvent by a suitable modification of the reaction energies. This can be achieved by using a continuum solvation model: the self-consistent continuum solvation model (SCCS) proposed by Andreussi, Dabo and Marzari ${ }^{36}$ and implemented in the Quantum espresso Environ tool ${ }^{31}$ has been adopted, giving an implicit description of the solvents (methanol in our case).

\section{Step 3: Oxidation}

This step consists in performing the oxidation of a species by changing its electronic configuration through a transformation of (one or two) $\mathrm{OH}$ groups into non-terminated $=\mathrm{O}$ groups (and eventually transforming the N-H group into a N-group). Such a chemical transformation involves the release of two hydrogen atoms in our implementation and is actuated with a probability calculated as in eq. (1) where the energies of the corresponding reaction are used. 
At any time, a random number of species is chosen and their oxidation is eventually realized. The released molecular hydrogen is assumed to consequently leave the simulation cell. One example of this step is schematically reproduced in figure 2-a. It is clear that the type of building monomers (DHI, IQ, MQ or NQ) is not conserved during this step, thus determining a modification of concentration of DHI and its redox forms along the polymerization process.

\section{Results and Discussion}

To demonstrate the capabilities of our computational tool, we emulated the polymerization process of a system as large as a cube of side $118.2 \AA$ containing an initial number of 10000 eumelanin monomeric units. All the monomers in the simulation box are initially in their non-oxidized form (i.e., DHI). The simulation cell contained as many as 165000 atoms. The simulation of the polymerization process required a multi-step procedure to be performed: i) first, the monomers have been placed in an initial arbitrary volume in a random fashion; ii) the system has been fully relaxed at constant temperature $\mathrm{T}=300 \mathrm{~K}$ and fixed ambient pressure using a Nosé-Hoover thermostat in order to reach the equilibrium values of volume and density. iii) The polymerization algorithm has then been finally implemented keeping the temperature constant $(\mathrm{T}=300 \mathrm{~K})$. It is important to acknowledge that the simulation box is subjected to periodic boundary conditions and the presence of solvent (methanol) is included implicitly through a modified dielectric constant and using the corresponding probabilities in all the steps of the polymerization algorithm described above.

The simulation lasted approximately $2.4 \cdot 10^{6}$ timesteps until the end of the polymerization process. An image of a polymerized sample is shown in figure. 3. The system in its polymerized configuration shows a mass density of $1.47 \mathrm{~g} / \mathrm{cm}^{3}$, in very good agreement with

eumelanin samples realized via analogous experimental procedures $\left(1.4-1.77 \mathrm{~g} / \mathrm{cm}^{3}\right) .{ }^{37-39}$ As a first result, we show the average molecular size as a function of the polymerization time in fig. 4. The green curve is the result of the average over three different trajectories obtained 
from unlike initial distributions of monomers. Starting from the monomodal initial state, the onset of chemical interactions between the molecules determines an initially slow increase of the average molecular weight due the relatively small amount of oxidized monomers available to react. When their quantity is sufficiently high, the average weight rapidly increases until reaching eventually a constant value $\simeq 3$ after $\cdot 10^{6}$ time steps. Subsequent dynamical evolution of the system does not lead to a considerable further increase, meaning that the polymerization process is mostly concluded. The converged finite value of the average molecular size provides evidence that eumelanin samples are mainly composed by smallweight molecular structures.

In this respect, the actual distribution of oligomers shown in figure 5 offers a deeper insight. The distribution of oligomers follows an exponential dependence, with a high concentration of monomers rapidly decreasing as a function of the molecular weight. Interestingly, a maximum oligomer size is found at around 37. This result is in remarkably good agreement with recent experimental data of matrix-assisted laser desorption/ionization mass spectrometry which revealed the possibility that large polymeric structures (up to 30 monomeric units) can be formed. ${ }^{20}$

We now proceed with a deeper analysis of the polymerized sample, looking at the microscopic features of the molecules. In particular, attention will be drawn onto the structural configurations of the single molecules and on their oxidation state. Following the terminology introduced in a recent review on the subject, ${ }^{10}$ we refer to the variety of possible structural conformations as the eumelanin molecular disorder..$^{2,16,40}$ Similarly, all the possible distributions of redox states within a molecule are generally named electronic disorder. In the following we will treat these aspects separately. As it will become clear, this theoretical framework represents a convenient scheme to tackle the problem allowing to develop meaningful physical insight. 


\section{Molecular disorder}

We now concentrate on the molecular disorder of eumelanins which is related to the variety of structures obtained by unlike assembling of the monomeric building blocks. The number of possible structures is extremely large, given that for each given oligomer size (from 1 to more than 40, see figure 5 ) very many different molecular configurations can be formed. For example, the number of possible tetramers can be roughly estimated: assuming four possible reactive sites in each monomer where binding with other units can occur, we obtain $4 \cdot 4 \cdot(4 \cdot 3) \cdot(4 \cdot 3) \cdot(1 / 2)=1152$ structural models (two of the four monomers bind at only one site, the remaining monomers at two sites). A systematic classification of all of them is unpracticable, yet useful information on the most stable and frequent structural configurations can be obtained by analyzing the polymerized samples already described. To this aim, we exploit the fact that each oligomer structure can be univocally identified by the sequence of chemical bonds linking each pair of connected monomers within the molecule. All the possible chemical bonds are shown figure 6 . We then identify the bonds which are more frequent in the polymerized samples described above. The results of this statistical analysis are shown in the same figure where we present the relative occurrence of each bond created at the end of the polymerization process. It is here evident how some bonds are generally more frequent than others, implying that they are energetically favored. Further analysis shows that such a distribution of bonds is not dissimilar to the ones obtained when considering oligomers with fixed size separately. Hence, this leads us to argue that such frequent bonds also have a positive impact on the overall molecular stability.

These results are consistent with experimental studies concerning the nature of different dimer, trimer or tetrameric oligomers. Thus, the 2,4' and 2,7' are the more frequent types of linkages observed in dimers ${ }^{20,41,42}$ although the 2,2 ' is also obtained under special conditions. ${ }^{41}$ The 2,4' and 2,7' linkages are still the more frequently observed in trimeric oligomers but the 3,3', 4,4' and 2,3' are also observed in tetrameric oligomers. ${ }^{43,44}$ Although this comparison should be taken with care because of the different conditions under which these 
studies were carried out, it clearly shows that the more prevalent types of linkages obtained in our study are consistent with the presently known experimental observations.

An interesting question arises about the presence of molecules whose structure is not strictly linear, in the sense that they contain cyclic sub-units. In all the samples considered, only "linear" molecules have been found. Although this does not rule out completely the possible formation of cyclic structures due to possible chemical reactions which were not considered, we argue that, upon the particular scheme of poymerization process assumed, non-strictly linear molecules are generally less frequent than linear ones.

\section{Electronic disorder}

Electronic disorder relates to the distribution of redox states within the eumelanin molecules or, equivalently, to the number and type of their constituing monomers (DHI, IQ, MQ, NQ). The basic monomers are shown in figure 1: they have the same backbone, but differ in the possible presence of $=\mathrm{O}$ groups in place of $-\mathrm{OH}$. Also the $\mathrm{N}-\mathrm{H}$ fragment can be replaced by a single $\mathrm{N}$ in the monomers. The different terminations of the monomeric units naturally determine different electronic configurations of the molecules, with a consequent change in the single or double bonds among the constituting atoms. It is clear that the number of possible electronic configurations is again remarkably large: e.g. for a tetramer there are four possible oxidation monomeric forms (including the DHI monomer), implying $4^{4}=256$ electronic configurations.

Nonetheless, it is important to acknowledge that (i) the distribution of redox states generally affects the overall molecule stability and (ii) the redox state of each monomer (and hence of a molecule) can change during the polymerization process. As for the latter, this possibility is properly implemented through the "oxidation" step in the polymerization algorithm (see figure 2 for instance). It is then interesting to analyze the evolution of the electronic state of the monomers (from now on we refer as monomers to both real monomers and the monomeric units within an oligomer) during the overall polymerization process. In this respect, figure 7 
shows how the relative concentrations of DHI, IQ, MQ, and NQs vary as a function of time. All the values are averaged over three independent realizations of the polymerization process. Starting from an initial condition where all monomers are in the DHI non-oxidized form, the number of DHI blocks exponentially decreases, transforming into one of its oxidized forms. The DHI concentration eventually reaches a stationary value smaller than 0.05 , which is the equilibrium result of two opposite yet related processes: the DHI creation from coupling and the DHI reduction due to monomer oxidation. MQs, NQs and IQs correspondingly increase. This demonstrates how the presence of $=\mathrm{O}$ groups in place of $-\mathrm{OH}$ can improve the stability of the molecules and favor the growth process.

However, this analysis does not allow us to characterize the redox state of the different oligomers. We thus look now at the concentration of non-terminated oxygen atoms in oligomers of different size. Figure 8 shows the average number of $=\mathrm{O}$ groups normalized with respect to the oligomer size (i.e. the average of $=\mathrm{O}$ groups performed over all the oligomers of a given size and divided by the size). Most of these values are not far from 1 . In particular the average over all sizes is found to be slightly larger than 1 (see dashed line in the figure), meaning that the number of $=\mathrm{O}$ groups increases almost linearly with the oligomer size. This leads us to conclude that, independently from the oligomer size, more frequent eumelanin molecules tend to have a moderately large number of non-terminated oxygen atoms. This result confirms a fortiori the pivotal role played by the electronic state in the eumelanin molecular stability, being the most frequent molecules also the most energetically stable.

\section{Conclusions}

A new computational approach merging molecular dynamics simulations and first-principles DFT calculations has been used to simulate the oxidative polymerization process of eumelamine in methanol at $300 \mathrm{~K}$. The approach combines both dynamical and chemical (oxidation and coupling of different oligomeric species) aspects in a fully atomistic iterative 
modeling of the process. The use of a probabilistic approach of the chemical steps is the key aspect in making such a fully atomistic modeling feasible. An exponential distribution of the oligomers molecular weight emerges and the average value is found to be lower than 10. The maximum oligomer size is found to be around 37. All these results are in good agreement with the available experimental information. The new approach provides also a convenient platform to characterize from a microscopic perspective the resulting eumelamin samples. In particular, we have performed a statistical analysis of the two sources of disorder, molecular and electronic, in the sample. The more frequent types of coupling between monomeric units in the final samples are shown to be consistent with experimental studies designed to characterize different dimeric, trimeric and tetrameric oligomers. The importance of oxidized $=\mathrm{O}$ groups in accelerating the growth process is also highlighted. Last but not least, the computational approach can be readily adapted to incorporate variations in the chemical processes behind the eumelanine growth. In view of the uncertainty concerning many aspects of the mechanism, the proposed approach may be an invaluable tool in testing variations of the mechanism, the influence of different parameters and solvents in the growth and ultimately in unravelling the key mechanistic aspects under different conditions.

\section{Acknowledgement}

We acknowledge the Regione Sardegna basic research Project No. CRP78744 "Energy Applications with Porous Silicon (ENAPSi)." E.C. acknowledges support by MINECO (Spain) through Grant FIS2015-64886-C5-4-P and the Severo Ochoa Centers of Excellence Program (Grant SEV-2015-0496) as well as by Generalitat de Catalunya (2014SGR301). 


\section{References}

(1) Ito, S.; Wakamatsu, K.; d'Ischia, M.; Napolitano, A.; Pezzella, A. Melanins and melanosomes; Wiley-VCH Verlag GmbH and Co. KGaA, 2011; pp 167-185.

(2) d'Ischia, M.; Napolitano, A.; Pezzella, A.; Meredith, P.; Sarna, T. Chemical and structural diversity in eumelanins: unexplored bio-optoelectronic materials. Angew. Chem. Int. Ed. 2009, 48, 3914-3921.

(3) Panzella, L.; Gentile, G.; D’Errico, G.; Della Vecchia, N. F.; Errico, M. E.; Napolitano, A.; Carfagna, C.; d'Ischia, M. Atypical structural and $\pi$-electron features of a melanin polymer that lead to superior free-radical-scavenging properties. Angew. Chem. Int. Ed. 2013, 52, 12684-12687.

(4) Bettinger, C. J.; Bruggeman, J. P.; Misra, A.; Borenstein, J. T.; Langer, R. Biocompatibility of biodegradable semiconducting melanin films for nerve tissue engineering. Biomaterials 2009, 30, 3050-3057.

(5) Pezzella, A.; Barra, M.; Musto, A.; Navarra, A.; Alfe, M.; Manini, P.; Parisi, S.; Cassinese, A.; Criscuolo, V.; d'Ischia, M. Stem cell-compatible eumelanin biointerface fabricated by chemically controlled solid state polymerization. Mater. Horiz. 2015, 2, $212-220$.

(6) Prasetyanto, E. A.; Manini, P.; Napolitano, A.; Crescenzi, O.; d'Ischia, M.; De Cola, L. Towards eumelanin zeolite hybrids: pore-size-controlled 5, 6-dihydroxyindole Polymerization. Chem. Eur. J. 2014, 20, 1597-1601.

(7) Shanmuganathan, K.; Cho, J. H.; Iyer, P.; Baranowitz, S.; Ellison, C. J. Thermooxidative stabilization of polymers using natural and synthetic melanins. Macromolecules 2011, 44, 9499-9507. 
(8) Antidormi, A.; Melis, C.; Canadell, E.; Colombo, L. Assessing the performance of Eumelanin/Si interface for photovoltaic applications. The Journal of Physical Chemistry C 2017, 121, 11576-11584.

(9) Pinna, E.; Melis, C.; Antidormi, A.; Cardia, R.; Sechi, E.; Cappellini, G.; d'Ischia, M.; Colombo, L.; Mula, G. Deciphering molecular mechanisms of interface buildup and stability in porous si/eumelanin hybrids. International Journal of Molecular Sciences 2017,18 .

(10) d'Ischia, M.; Napolitano, A.; Ball, V.; Chen, C.-T.; Buehler, M. J. Polydopamine and eumelanin: from structure-property relationships to a unified tailoring strategy. Acc. Chem. Res. 2014, 47, 3541-3550.

(11) Liebscher, J.; Mrówczyński, R.; Scheidt, H. A.; Filip, C.; Hadade, N. D.; Turcu, R.; Bende, A.; Beck, S. Structure of polydopamine: a never-ending story? Langmuir 2013, 29, 10539-10548.

(12) Lin, S.; Chen, C.-T.; Bdikin, I.; Ball, V.; Grácio, J.; Buehler, M. J. Tuning heterogeneous poly (dopamine) structures and mechanics: in silico covalent cross-linking and thin film nanoindentation. Soft Matter 2014, 10, 457-464.

(13) Dreyer, D. R.; Miller, D. J.; Freeman, B. D.; Paul, D. R.; Bielawski, C. W. Elucidating the structure of poly (dopamine). Langmuir 2012, 28, 6428-6435.

(14) Cheng, J.; Moss, S. C.; Eisner, M.; Zschack, P. X-Ray characterization of melanins I. Pigm. Cell Res. 1994, 7, 255-262.

(15) Chen, C.-T.; Chuang, C.; Cao, J.; Ball, V.; Ruch, D.; Buehler, M. J. Excitonic effects from geometric order and disorder explain broadband optical absorption in eumelanin. Nat. Commun. 2014, 5 . 
(16) Meredith, P.; Powell, B. J.; Riesz, J.; Nighswander-Rempel, S. P.; Pederson, M. R.; Moore, E. G. Towards structure-property-function relationships for eumelanin. Soft Matter 2006, 2, 37-44.

(17) Zajac, G.; Gallas, J.; Cheng, J.; Eisner, M.; Moss, S.; Alvarado-Swaisgood, A. The fundamental unit of synthetic melanin: a verification by tunneling microscopy of X-ray scattering results. Biochim. Biophys. Acta, Gen. Subj. 1994, 1199, 271-278.

(18) Zajac, G.; Gallas, J.; Alvarado-Swaisgood, A. Tunneling microscopy verification of an xray scattering-derived molecular model of tyrosine-based melanin. J. Vac. Sci. Technol., $B$ 1994, 12, 1512-1516.

(19) Díaz, P.; Gimeno, Y.; Carro, P.; González, S.; Schilardi, P. L.; Benítez, G.; Salvarezza, R. C.; Creus, A. H. Electrochemical self-assembly of melanin films on gold. Langmuir 2005, 21, 5924-5930.

(20) Reale, S.; Crucianelli, M.; Pezzella, A.; d'Ischia, M.; De Angelis, F. Exploring the frontiers of synthetic eumelanin polymers by high-resolution matrix-assisted laser/desorption ionization mass spectrometry. Journal of Mass Spectrometry 2011, $47,49-53$.

(21) Okuda, H.; Wakamatsu, K.; Ito, S.; Sota, T. Possible oxidative polymerization mechanism of 5,6-dihydroxyindole from ab initio calculations. The Journal of Physical Chemistry A 2008, 112, 11213-11222.

(22) Liu, H.; Li, H.; Lu, Z.-Y. Incorporating chemical reactions in dissipative particle dynamics simulations. Procedia Computer Science 2011, 4, 1021-1028.

(23) Yong, X.; Kuksenok, O.; Balazs, A. C. Modeling free radical polymerization using dissipative particle dynamics. Polymer 2015, 72, 217 - 225. 
(24) Shao, J.; Tang, W.; Xia, R.; Feng, X.; Chen, P.; Qian, J.; Song, C. Monte Carlo simulation on kinetics of batch and semi-batch free radical polymerization. Macromolecular Research 2015, 23, 1042-1050.

(25) D'hooge, D. R.; Steenberge, P. H. V.; Reyniers, M.-F.; Marin, G. B. The strength of multi-scale modeling to unveil the complexity of radical polymerization. Progress in Polymer Science 2016, 58, 59 - 89, Topical Volume Polymer Chemistry.

(26) Plimpton, S. Fast parallel algorithms for short-range molecular dynamics. Journal of Computational Physics 1995, 117, $1-19$.

(27) Ponder, J. W.; Case, D. A. Protein simulations; Advances in Protein Chemistry; Academic Press, 2003; Vol. 66; pp $27-85$.

(28) Genovese, C.; Antidormi, A.; Dettori, R.; Caddeo, C.; Mattoni, A.; Colombo, L.; Melis, C. Linking morphology to thermal conductivity in PEDOT: an atomistic investigation. Journal of Physics D: Applied Physics 2017, 50, 494002.

(29) Wang, J.; Wolf, R. M.; Caldwell, J. W.; Kollman, P. A.; Case, D. A. Development and testing of a general amber force field. Journal of computational chemistry 2004, 25, $1157-1174$.

(30) d'Ischia, M.; Napolitano, A.; Pezzella, A.; Land, E. J.; Ramsden, C. A.; Riley, P. A. In 5,6-Dihydroxyindoles and indole-5,6-diones; Katritzky, A. R., Ed.; Advances in Heterocyclic Chemistry; Academic Press, 2005; Vol. 89; pp 1 - 63.

(31) Giannozzi, P. et al. QUANTUM ESPRESSO: a modular and open-source software project for quantum simulations of materials. J. Phys.-Condens. Mat. 2009, 21, 395502.

(32) Perdew, J. P.; Burke, K.; Ernzerhof, M. Generalized gradient approximation made simple. Phys. Rev. Lett. 1996, 77, 3865-3868. 
(33) Vanderbilt, D. Soft self-consistent pseudopotentials in a generalized eigenvalue formalism. Phys. Rev. B 1990, 41, 7892-7895.

(34) Grimme, S. Semiempirical GGA-type density functional constructed with a long-range dispersion correction. J. Comput. Chem. 2006, 27, 1787-1799.

(35) Barone, V.; Casarin, M.; Forrer, D.; Pavone, M.; Sambi, M.; Vittadini, A. Role and effective treatment of dispersive forces in materials: polyethylene and graphite crystals as test cases. J. Comput. Chem. 2009, 30, 934-939.

(36) Andreussi, O.; Dabo, I.; Marzari, N. Revised self-consistent continuum solvation in electronic-structure calculations. J. Chem. Phys. 2012, 136, 064102.

(37) Gallas, J.; Littrell, K.; Seifert, S.; Zajac, G.; Thiyagarajan, P. Solution structure of copper ion-induced molecular aggregates of tyrosine melanin. Biophysical Journal 1999, $77,1135-1142$.

(38) Zeise, L.; Addison, R. B.; Chedekel, M. R. Bio-analytical studies of Eumelanins. I. Characterization of melanin the particle. Pigment Cell Research 1990, 3, 48-53.

(39) Moses, D.; Mattoni, M.; Slack, N.; Waite, J.; Zok, F. Role of melanin in mechanical properties of Glycera jaws. Acta Biomaterialia 2006, 2, 521 - 530 .

(40) Tran, M. L.; Powell, B. J.; Meredith, P. Chemical and structural disorder in eumelanins: a possible explanation for broadband absorbance. Biophys. J. 2006, 90, 743-752.

(41) Napolitano, A.; Corradini, M. G.; Prota, G. A reinvestigation of the structure of melanochrome. Tetrahedron Letters 1985, 26, $2805-2808$.

(42) d'Ischia, M.; Napolitano, A.; Tsiakas, K.; Prota, G. New intermediates in the oxidative polymerisation of 5,6-dihydroxyindole to melanin promoted by the peroxidase/H2O2 system. Tetrahedron 1990, 46, 5789-5796. 
(43) Panzella, L.; Pezzella, A.; Napolitano, A.; d'Ischia, M. The first 5,6-dihydroxyindole tetramer by oxidation of 5,5',6,6'-tetrahydroxy- 2,4'-biindolyl and an unexpected issue of positional reactivity en route to eumelanin-related polymers. Organic Letters 2007, 9, 1411-1414.

(44) Pezzella, A.; Panzella, L.; Natangelo, A.; Arzillo, M.; Napolitano, A.; d'Ischia, M. 5,6-dihydroxyindole tetramers with "anomalous" interunit bonding patterns by oxidative coupling of 5,5',6,6'-tetrahydroxy-2,7'-biindolyl: emerging complexities on the way toward an improved model of eumelanin buildup. The Journal of Organic Chemistry 2007, 72, 9225-9230. 



Figure 1: Monomeric species involved in eumelanins that are considered in the present work: 5,6-dihydroxy-indole (DHI) and its three redox forms (indolequinone (IQ), quinone-methide (MQ) and quinone-imine (NQ)). The labelling used for the different C positions is shown in the DHI molecule. 
a)



b)



Figure 2: Schematic examples of the "oxidation" and "coupling" steps occurring in the eumelanin polymerization. (a) (oxidation) A DHI monomer transforms into its IQ form (formally releasing two hydrogen atoms). (b) (coupling) A DHI monomer covalently bonds with an IQ unit producing an unoxidized dimer. 
a)



b)

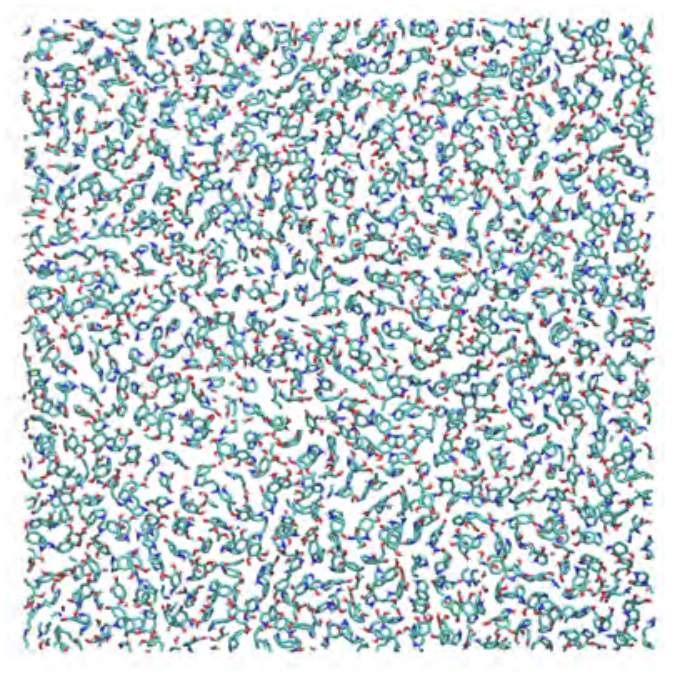

Figure 3: Image of the eumelanin sample at the end of the polymerization process. a) 3D view, b) section view 


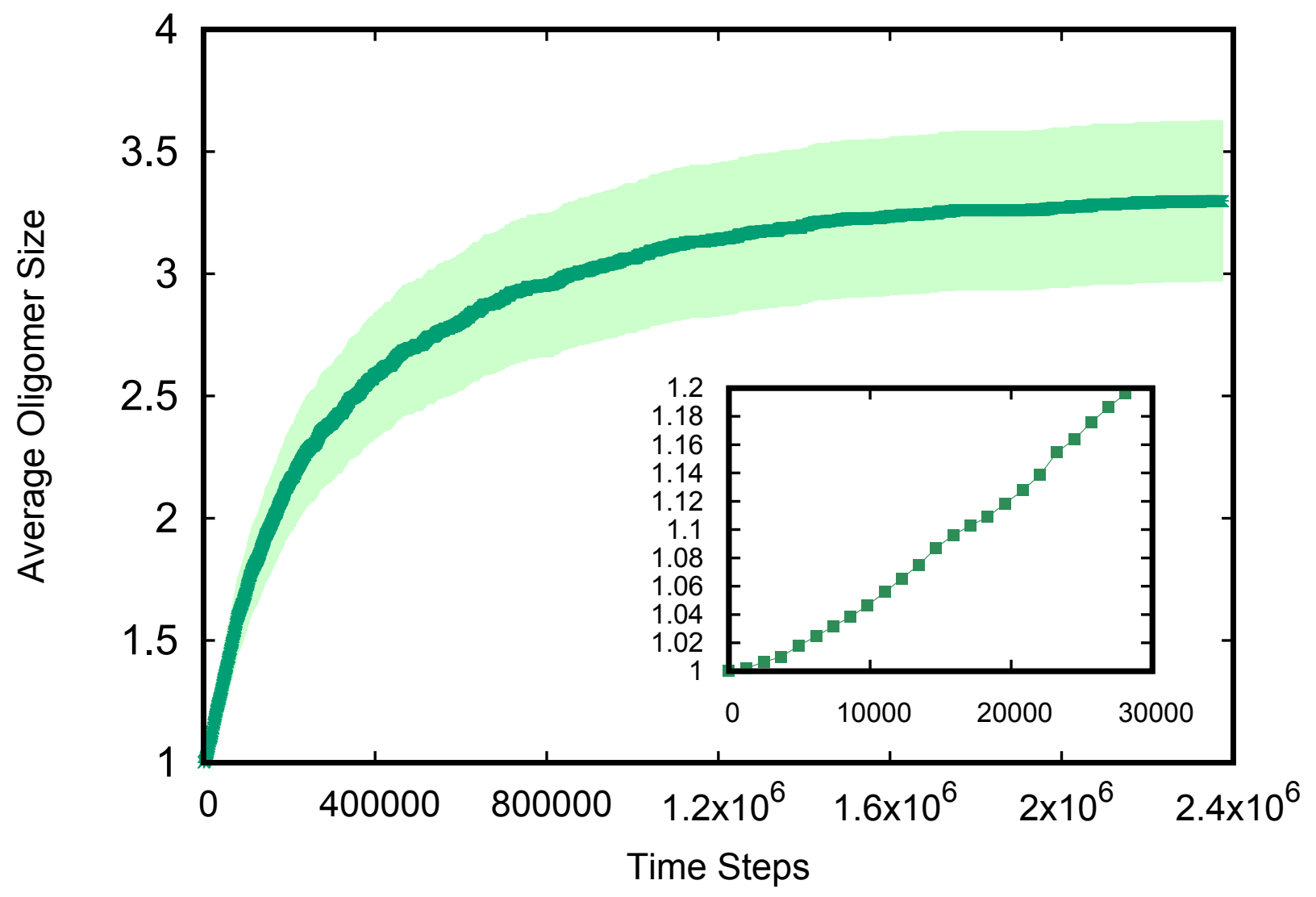

Figure 4: Average oligomer size as a function of time. The green curve is the average over three different initial configurations of the system. The corresponding uncertainty is shown by the shadowed area. In the inset, the slow increase found at the onset of the polymerization process. 


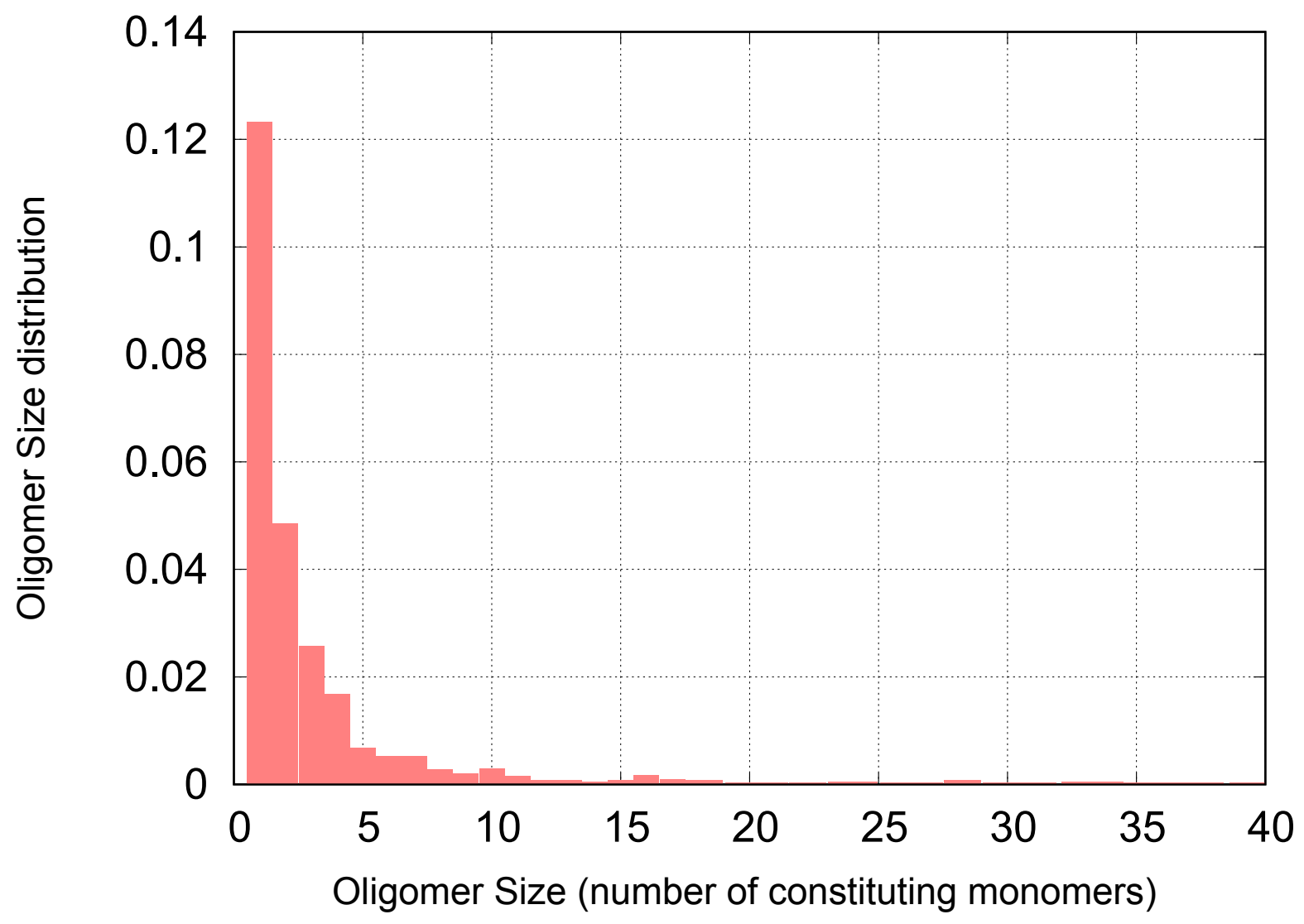

Figure 5: Statistical distribution of the average molecular size in the eumelanin samples at the end of polymerization. An exponential dependence is found. 
BOND



$3,3^{\prime}$

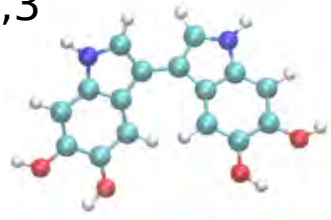

$7,7^{\prime}$

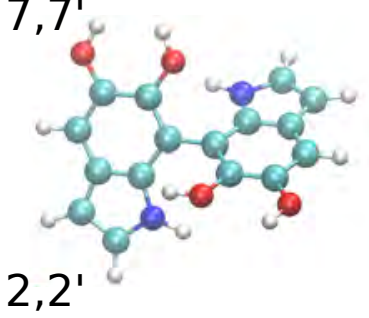

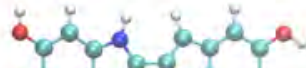
arab gato
FREQUENCY

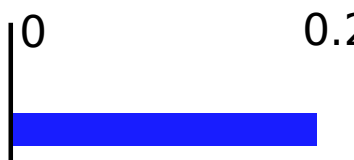

0.17
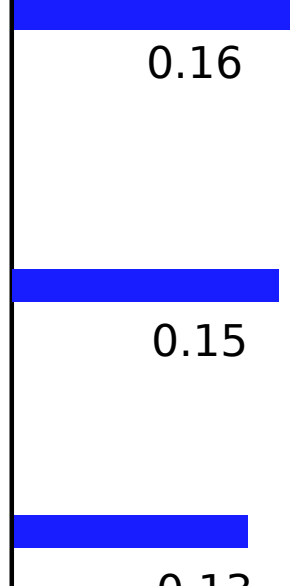

0.13

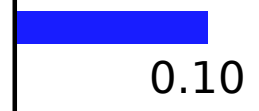

BOND

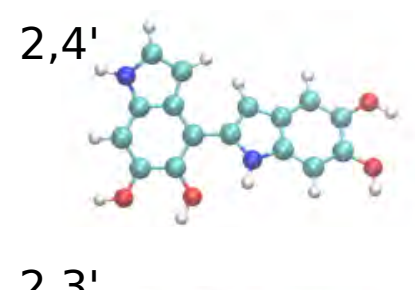

$2,3^{\prime}$

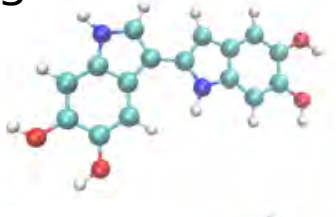

$3,7^{\prime}$

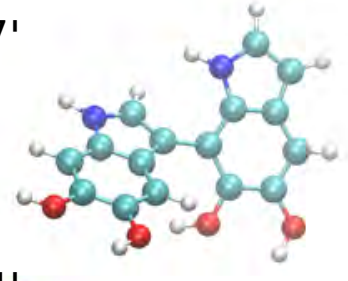

3,4

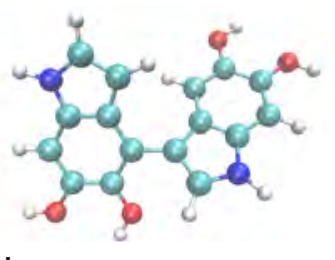

$4,7^{\prime}$

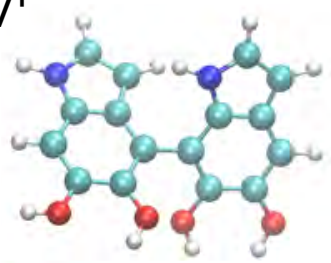

FREQUENCY

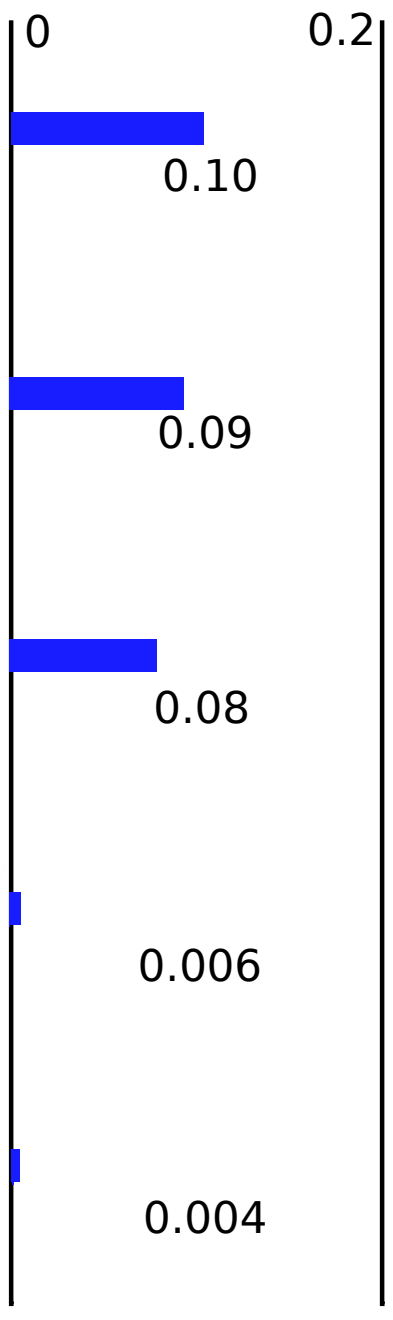

Figure 6: Statistical analysis of the chemical bonds in the polymerized eumelanin samples. From top to bottom, the most frequent chemical bonds are shown. The molecular plots are only meant to illustrate the $n, m$ ' type of coupling independently of the redox states. 


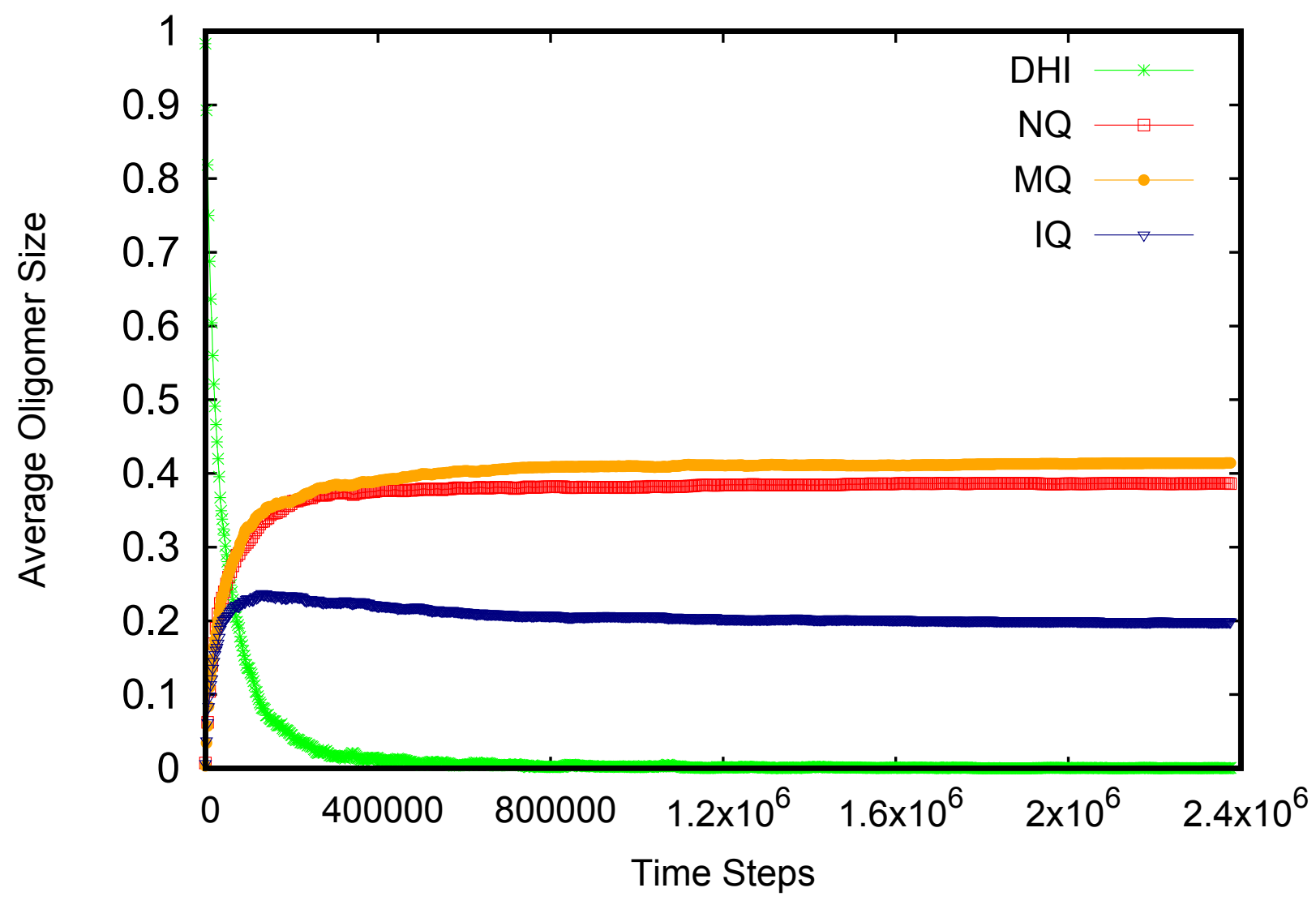

Figure 7: Relative concentration of DHI and its redox forms during the polymerization process. The values are averaged over three different realizations of the system evolution. The gradual reduction of DHI units is due to their oxidation into IQ, MQ and NQ monomers. 


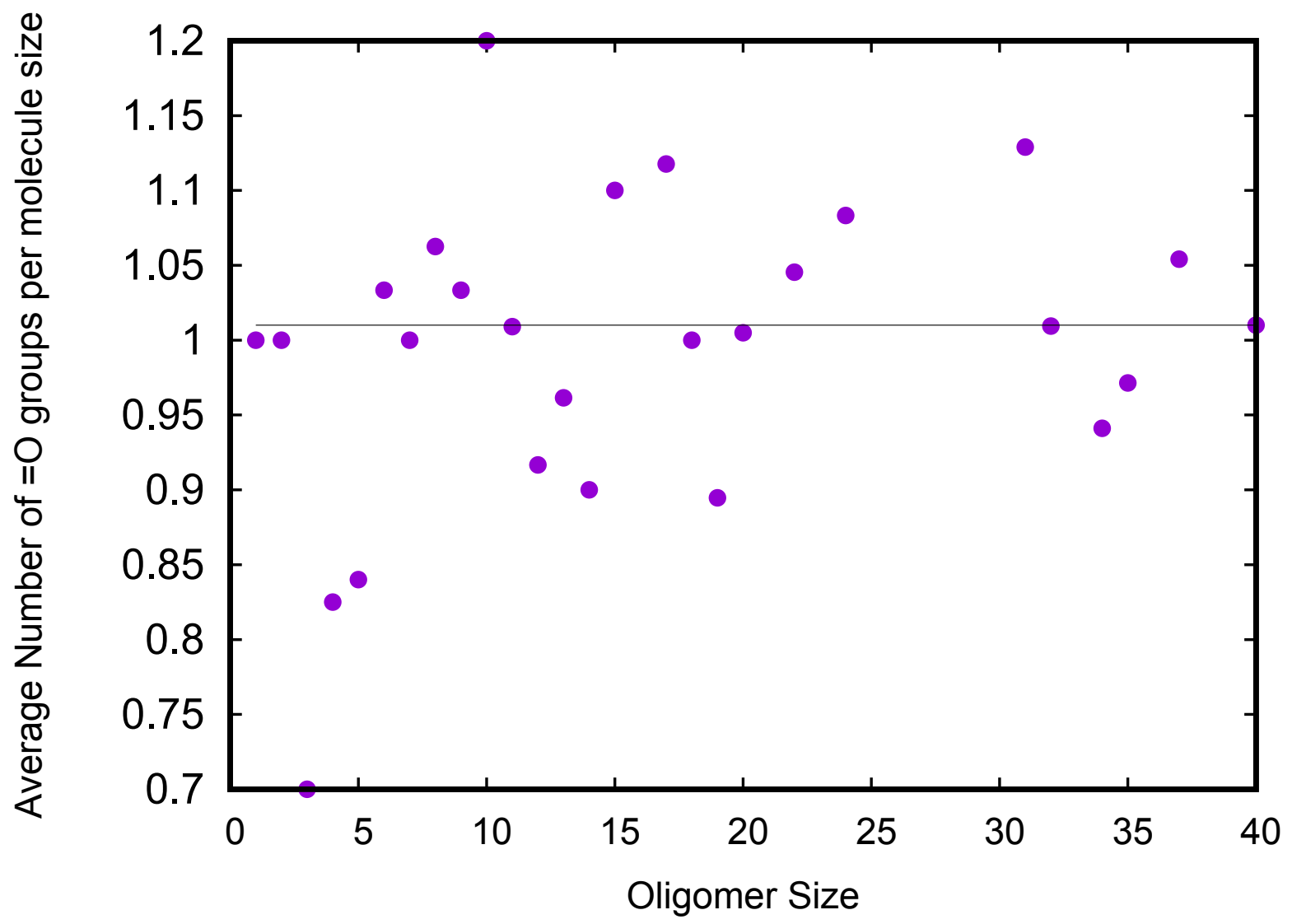

Figure 8: Average number of $=\mathrm{O}$ groups in the eumelanin sample performed over all the oligomers of a given size and divided by the size (purple points). The dashed line is the mean value. 


\section{Graphical TOC Entry}

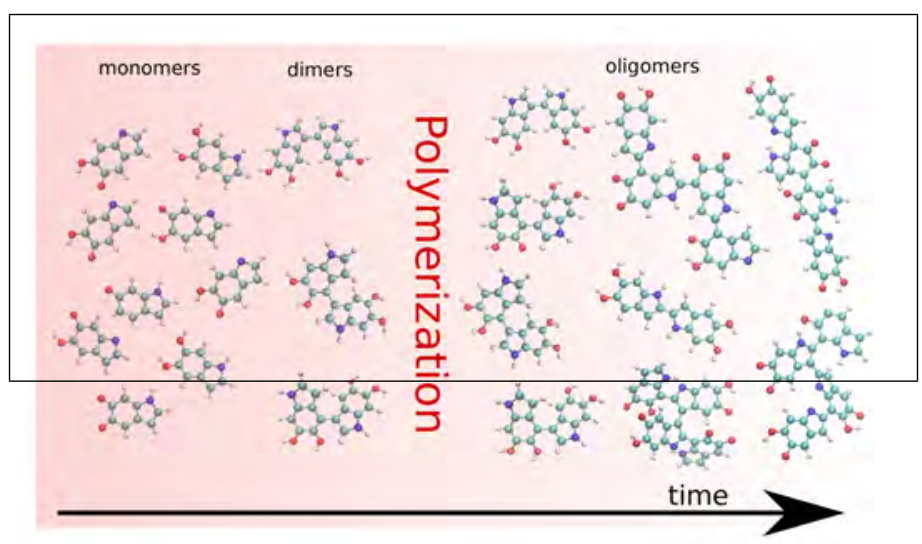

